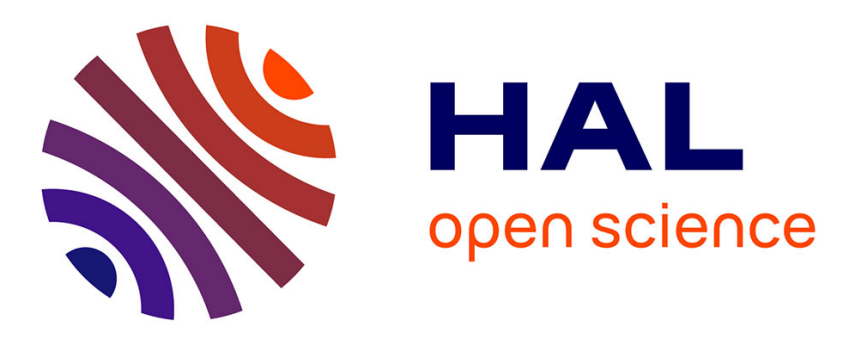

\title{
Stochastic non-linear response of a flexible rotor with local non-linearities
}

\author{
Jean-Jacques Sinou, J Didier, B Faverjon
}

\section{To cite this version:}

Jean-Jacques Sinou, J Didier, B Faverjon. Stochastic non-linear response of a flexible rotor with local non-linearities. International Journal of Non-Linear Mechanics, 2015, 74, pp.92 - 99. 10.1016/j.ijnonlinmec.2015.03.012 . hal-03258030

\section{HAL Id: hal-03258030 https://hal.science/hal-03258030}

Submitted on 11 Jun 2021

HAL is a multi-disciplinary open access archive for the deposit and dissemination of scientific research documents, whether they are published or not. The documents may come from teaching and research institutions in France or abroad, or from public or private research centers.
L'archive ouverte pluridisciplinaire HAL, est destinée au dépôt et à la diffusion de documents scientifiques de niveau recherche, publiés ou non, émanant des établissements d'enseignement et de recherche français ou étrangers, des laboratoires publics ou privés. 


\title{
Stochastic nonlinear response of a flexible rotor with local
}

\section{nonlinearities}

\author{
J-J. Sinou ${ }^{a}$, J. Didier ${ }^{a}$ and B. Faverjon ${ }^{b}$ \\ ${ }^{a}$ Laboratoire de Tribologie et Dynamique des Systèmes UMR 5513, Ecole Centrale de Lyon, France. \\ ${ }^{b}$ Laboratoire de Mécanique des Contacts et des Structures UMR 5259, INSA Lyon, France.
}

\begin{abstract}
The effects of uncertainties on the non linear dynamics response remains misunderstood and most of the classical stochastic methods used in the linear case fail to deal with a non linear problem. So we propose to take into account of uncertainties into non linear models, by coupling the Harmonic Balance Method (HBM) and the Polynomial Chaos Expansion (PCE). The proposed method called the Stochastic Harmonic Balance Method (Stochastic-HBM) is based on a new formulation of the non linear dynamic problem in which not only the approximated nonlinear responses but also the nonlinear forces and the excitation pulsation are considered as stochastic parameters. Expansions on the PCE basis is performed by passing via an Alternate Frequency Time method with Probabilistic Collocation (AFTPC) for estimating the stochastic non linear forces in the stochastic domain and the frequency domain. In the present paper, the Stochastic Harmonic Balance Method (Stochastic-HBM) is applied to a flexible non-linear rotor system, with random parameters modeled as random fields is presented. The Stochastic-HBM combined with an Alternate Frequency-Time method with Probabilistic Collocation (AFTPC) allows to solve dynamical problems with non-regular non linearities in presence of uncertainties. In this study, the procedure is developed for the estimation of stochastic nonlinear responses of the rotor system with different regular and non-regular non-linearities. The finite element rotor system is composed of a shaft with two disks and two flexible bearing supports where the non-linearities are due to a radial clearance or a cubic stiffness. A numerical analysis is performed to analyze the effect of uncertainties on the non-linear behavior of this rotor system by using the Stochastic-HBM. Furthermore, the results are compared with those obtained by applying a classical Monte-Carlo simulation to demonstrate the efficiency of the proposed methodology.
\end{abstract}

\section{Introduction}

During the past decade, numerous studies have been done to understand and to model the non linear phenomenon structural dynamics. Most of these models deal with determinist parameters. But in the design of many types of engineering systems, geometrical, materials and non linear parameters are often uncertain due to the manufacturing process for example. So an expected improvement and an obvious extension of the classical deterministic studies are the consideration of uncertainties when designing a system according to certain operating conditions. However, the effects of uncertainties on the non linear dynamics response of mechanical systems remains misunderstood and most of the classical stochastic methods used in the linear case fail to deal with a non linear problem. This is especially true in the context of designing rotating machines [1, 2, 3].

Numerous methods have been developed to take into account uncertainties in the dynamic response: the classical perturbation methods, the Monte Carlo method or the Polynomial Chaos Expansion for example. The perturbation methods are based on the development of random quantities in Taylor or Neumann series $[4,5,6]$. These methods have proved their effectiveness for 
small variations. One of the most classical method is Monte Carlo Simulations (MCS) which include uncertainties by generating samples of the random parameter in the deterministic model. This method can be used to deal with a linear or non-linear problem but MCS have a high computational cost due to the fact that a high number of samples is necessary to reach the converged response of the mechanical system under study. To reduce the computational cost, one of the most useful methods is the Polynomial Chaos Expansion (PCE) [7, 8]. This method has proved its robustness and efficiency on linear dynamic problems (and more specifically for analyzing the dynamics of rotating machines $[9,10,11,12])$ but PCE does not seem adapted to deal with multiples solutions observed in non linear systems.

So we propose to take into account uncertainties into non linear rotor models by applying a novel nonlinear method called the Stochastic Harmonic Balance Method (Stochastic-HBM [13]). This approach that is an extension of the Harmonic Balance Method (HBM) that is one of the mathematical approaches to solve deterministic equations with non linear terms $[14,15,16$, 17, 18, 19] and the Polynomial Chaos Expansion (PCE) that is one of the most used stochastic parametric method [7, 8]. In addition an Alternating Frequency Time method [20] with Probabilistic Collocation (PC) for calculating the stochastic non linear forces in stochastic domain and frequency domain is proposed. This approach proposes also a new formulation of the non linear stochastic dynamic problem in which the excitation pulsation is considered as a stochastic parameter and expanded on the PCE basis. Numerical simulations for a simple nonlinear two-degree-of-freedom model with different types of nonlinearities were carried-out to appraise the global methodology. Considering uncertainties in linear and nonlinear parts of the mechanical system, it was demonstrated that the quasi-periodic stochastic dynamic response evaluated by this approach agreed very well with those obtained from the classical Monte Carlo Simulation. Moreover, it is found that the results obtained via the Stochastic-HBM require significantly less computation.

So the objective of the present study is to demonstrate efficiencies and strong capabilities of this numerical approach to calculate an accurate stochastic response of large rotating systems and to discuss the need to consider both nonlinear terms and uncertainties in rotating machines in order to obtain an accurate solution in a design process.

The paper is organized as follows: firstly, the non-linear rotor system with uncertainty is presented. Then, the process to calculate the dynamic response of the stochastic rotor model is presented by detailing the Stochastic-HBM combined with the Alternate Frequency-Time method and the Probabilistic Collocation. Finally, numerical simulations are proposed in order to illustrate the main capabilities of the proposed methodology and to investigate the influence of both nonlinearities and uncertainties for a flexible rotor system.

\section{Rotor system with uncertainties}

\subsection{General equation of the rotor system}

The rotor system under study is consists of a shaft with two discs. The shaft is discretized into 10 Euler beam finite elements with four degrees of freedom at each node (two lateral displacements and two rotations, the axial and torsional degrees of freedom being not considered). Two bearing supports are added at each end of the rotor. All the material properties and dimensions of the rotor are given in Table 1. The layout of the rotor system is shown in Figures 1(a) and the nodal displacement vector defined as $\left[\begin{array}{llllllll}v_{1} & w_{1} & \theta_{1} & \psi_{1} & v_{2} & w_{2} & \theta_{2} & \psi_{2}\end{array}\right]$ in fixed frame are given in Figure 1(b).

After assembling the different elements of the rotor [21, 3], we obtain the equation of motion of this rotor system

$$
\mathbf{M} \ddot{\mathbf{x}}(t)+(\mathbf{C}+\omega \mathbf{G}) \dot{\mathbf{x}}(t)+\mathbf{K x}(t)=\mathbf{f}(t)+\mathbf{f}_{\mathbf{n l}}(\mathbf{x}(t))
$$

with $\mathbf{M}, \mathbf{K}$ and $\mathbf{G}$ are the mass, stiffness and gyroscopic matrices of the complete system (the shaft, the two rigid disc and the 
two bearing supports), respectively. Matrices expressions can be found in [9]. C defines the matrix of Rayleigh damping of the shaft. $\omega$ is the rotational speed of the shaft. $\mathbf{f}$ corresponds to the linear forces (i.e. gravitational forces and unbalance forces) applied to the rotor system. $\mathbf{f}_{\mathbf{n l}}$ contains the nonlinear forces. The non linearity is located only on the first bearing. Two kinds of nonlinear forces have been conducted in order to demonstrate the efficiency and robustness of the proposed approach:

- a regular cubic polynomial non-linearity. The restoring force at the first bearing support for both the $x$-direction and $y$-direction are given by

$$
\left\{\begin{array}{l}
f_{n l_{x}}(t)=-k_{n l} v^{3}(t) \\
f_{n l_{y}}(t)=-k_{n l} w^{3}(t)
\end{array}\right.
$$

where $k_{n l}$ defines the additional nonlinear term. $v$ and $w$ are the relative relative displacements in the $x$-direction and $y$-direction, as illustrated in Figure 1(b)

- a non-regular non-linearity with contact and no-contact states. The restoring force can be estimated by the following relation

$$
\left\{\begin{array}{l}
f_{n l_{x}}(t)=-k_{1} v(t) \quad \text { if } \quad r(t) \leq r_{l i m} \\
f_{n l_{y}}(t)=-k_{1} w(t) \quad \text { if } \quad r(t) \leq r_{l i m} \\
f_{n l_{x}}(t)=\left(-k_{2}\left(r-r_{l i m}\right)-k_{1} r_{l i m}\right) \cos \alpha \quad \text { if } \quad r(t)>r_{l i m} \\
f_{n l_{y}}(t)=\left(-k_{2}\left(r-r_{l i m}\right)-k_{1} r_{l i m}\right) \sin \alpha \quad \text { if } \quad r(t)>r_{l i m}
\end{array}\right.
$$

with

$$
r=\sqrt{v^{2}+w^{2}} \quad ; \quad \cos \alpha=\frac{v}{r} \quad ; \quad \sin \alpha=\frac{w}{r}
$$

$k_{1}$ and $k_{2}$ define the effective stiffnesses before and after the contact state. $r_{l i m}$ represents the clearance value. $v$ and $w$ represents the nodal displacements along the $x$ and $y$ directions, as mentioned in Figure 1(a).

\subsection{Uncertainties in the rotor system}

In this paper, it has been chosen to investigate the effects of uncertainties by considering four specific uncertain parameters:

- the Young modulus of the shaft is taken as random and defined by following

$$
\tilde{E}=\bar{E}\left(1+\delta_{E} \xi_{1}\right)
$$

where $\bar{E}$ is the mean value of the of Young modulus of the shaft, $\xi_{1}$ is a random variable which is a standard normal deviate and, $\delta_{E}$ is the variation coefficient of the random Young modulus. From a practical point of view, it can be noted that the assumption of Gaussian Young's modulus is consistent with the small uncertainty used in the following numerical example.

- the nonlinearity of contact and no-contact states is supposed to be random: we consider the parameter $\tilde{k_{2}}$ that defines the effective stiffness of the system in contact state as stochastic:

$$
\tilde{k_{2}}=\overline{k_{2}}\left(1+\delta_{k_{2}} \xi_{2}\right)
$$


where $\overline{k_{2}}$ is the mean of stiffness in contact state, $\xi_{2}$ is a random variable which is a standard normal deviate and, $\delta_{k_{2}}$ is the variation coefficient of the effective stiffness.

- the nonlinear cubic term is supposed to be random: we define a random nonlinear stiffness $\tilde{k_{n l}}$ following defines the effective stiffness of the system in contact state as stochastic:

$$
\tilde{k_{n l}}=\overline{k_{n l}}\left(1+\delta_{k_{n l}} \xi_{3}\right)
$$

where $\overline{k_{n l}}$ is the mean value of the nonlinear stiffness, $\xi_{3}$ is a random variable which is a standard normal deviate, $\delta_{k_{n l}}$ is the variation coefficient of the nonlinear stiffness.

- the unbalance excitation is considered as a random quantity for the mass unbalance $\tilde{m}$ such as defines the effective stiffness of the system in contact state as stochastic:

$$
\tilde{m}=\bar{m}\left(1+\delta_{m} \xi_{4}\right)
$$

where $\bar{m}$ is the mean value of the mass unbalance, $\xi_{4}$ is a random variable which is a standard normal deviate, $\delta_{m}$ is the variation of the unbalance intensity.

[Figure 1 about here.]

\section{Stochastic response of the system based on the Stochastic Harmonic Balance Method}

In this section, the Stochastic Harmonic Balance Method will be briefly described. For more details, the reader is referred to the following paper [13].

The general equation of motion for the rotor system with nonlinearities and uncertainties can be written in the following form

$$
\tilde{\mathbf{M}}(\tau) \ddot{\tilde{\mathbf{x}}}(\tau, t)+(\tilde{\mathbf{C}}(\tau)+\tilde{\omega}(\tau) \tilde{\mathbf{G}}(\tau)) \dot{\tilde{\mathbf{x}}}(\tau, t)+\tilde{\mathbf{K}}(\tau) \tilde{\mathbf{x}}\left((\tau, t)=\tilde{\mathbf{f}}(\tau, t)+\tilde{\mathbf{f}_{\mathbf{n l}}}(\tau, t, \mathbf{x}(\tau, t))\right.
$$

where $\tau$ designates the random character and $\tilde{\mathbf{x}}(\tau, t)$ defines the non-linear stochastic response of the rotor.

Considering an extension of the deterministic harmonic balance method [16], we assume that the non-linear stochastic response of the rotor can be approximated by finite Fourier series of order $m$

$$
\tilde{\mathbf{x}}(\tau, t)=\tilde{\mathbf{A}_{0}}(\tau)+\sum_{j=1}^{m}\left(\tilde{\mathbf{A}_{j}}(\tau) \cos (j \tilde{\omega}(\tau) t)+\tilde{\mathbf{B}_{j}}(\tau) \sin (j \tilde{\omega}(\tau) t)\right)
$$

$\tilde{\mathbf{A}_{0}}(\tau), \tilde{\mathbf{A}_{j}}(\tau)$ and $\tilde{\mathbf{B}_{j}}(\tau)$ (with $j=1, \cdots, m$ ) define the unknown stochastic coefficients of the finite Fourier series. $\tilde{\omega}(\tau)$ defines the unknown stochastic frequency of the rotor system. At this stage, it is important to note that the approximated solutions $\tilde{\mathbf{x}}(\tau, t)$ have to include an unknown stochastic formulation for $\tilde{\omega}(\tau)$ in order to describe the possible evolution of the frequency of the rotor system. This is due to the potential presence of returning points, phenomena of reversal curve of the Frequency Response Function and the dependence of the frequency with amplitude of the system [13]. In the case of a system having only parametric terms (such as asymmetry, bow or misalignment for example), the previous expression (10) can be simplified by using the deterministic frequency $\omega$ that corresponds to the rotational speed of the rotor system. For more details the reader is referred to the following papers $[12,9,22]$. The number of harmonic coefficients $m$ is selected on the basis of the number of significant harmonics expected in the stochastic dynamical response of the rotor system. Techniques for the selection of the optimal number of harmonics have been proposed previously in the paper [17]. 
Then, it may be observed that the linear terms $\mathbf{f}$ including the gravitational forces and unbalance forces can also be exactly defined by finite stochastic Fourier series (with constant components and first-order periodic components in the frequency domain, respectively). Expressions are given by

$$
\tilde{\mathbf{f}}(\tau, t)=\tilde{\mathbf{C}}_{0}^{f}(\tau)+\tilde{\mathbf{C}}_{1}^{f}(\tau) \cos (\tilde{\omega}(\tau) t)+\tilde{\mathbf{S}}_{1}^{f}(\tau) \sin (\tilde{\omega}(\tau) t)
$$

Moreover, we assume that the nonlinear forces $\mathbf{f}_{\mathrm{nl}}$ can also be approximated by considering truncated stochastic Fourier series of order $m$

$$
\tilde{\mathbf{f}_{\mathbf{n l}}}(\tau, t)=\tilde{\mathbf{C}}_{0}^{f n l}(\tau)+\sum_{j=1}^{m}\left(\tilde{\mathbf{C}}_{j}^{f n l}(\tau) \cos (j \tilde{\omega}(\tau) t)+\tilde{\mathbf{S}}_{j}^{f n l}(\tau) \sin (j \tilde{\omega}(\tau) t)\right)
$$

The choice of the same order for the truncated series (i.e. the number of significant harmonic coefficients) for both the stochastic nonlinear response (see equation (10)) and the stochastic nonlinear forces (see equation (12)) is only due to the simplicity and ease in the formulation. The generalization for different orders of stochastic nonlinear responses and forces is immediate and without any difficulty for the numerical processing. The only difficulty may be the calculation of the stochastic Fourier coefficients $\tilde{\mathbf{C}}_{0}^{f n l}(\tau), \tilde{\mathbf{C}}_{j}^{f n l}(\tau)$ and $\tilde{\mathbf{S}}_{j}^{f n l}(\tau)$. This numerical process will be explained later in the paper.

In a general context where several excitations and materials parameters can be considered as random, the following random quantities have to be considered : mass, damping, gyroscopic and rigidity parameters (defined respectively by $\tilde{\mathbf{M}}(\tau), \tilde{\mathbf{C}}(\tau), \tilde{\mathbf{G}}(\tau)$ and $\tilde{\mathbf{K}}(\tau)$ ) as well as the linear and non linear excitation terms (defined respectively by $\tilde{\mathbf{f}}(\tau, t)$ and $\tilde{\mathbf{f}_{\mathbf{n l}}}(\tau, t)$ ). For the non-linear rotor system, the equations of motion (9) may be written in the stochastic domain as

$$
\left(\tilde{\mathbf{I 0}}(\tau)+\tilde{\omega}(\tau) \tilde{\mathbf{I}} \mathbf{1}(\tau)+\tilde{\omega}^{2}(\tau) \tilde{\mathbf{I}}(\tau)\right) \tilde{\mathbf{X}}(\tau)=\tilde{\mathbf{F}}(\tau)+\tilde{\mathbf{F}_{\mathbf{n l}}}(\tau)
$$

where $\tilde{\mathbf{X}}(\tau), \tilde{\mathbf{F}}(\tau)$ and $\tilde{\mathbf{F}} \tilde{\mathbf{n l}}(\tau)$ denote the unknown vector of the stochastic harmonic coefficients (i.e. $\tilde{\mathbf{A}_{0}}(\tau), \tilde{\mathbf{A}_{j}}(\tau)$ and $\tilde{\mathbf{B}_{j}}(\tau)$ with $j=1, \cdots, m$, see equation (10)), the projection of the linear forces (i.e. $\tilde{\mathbf{C}}_{0}^{f}(\tau), \tilde{\mathbf{C}}_{1}^{f}(\tau)$ and $\tilde{\mathbf{S}}_{1}^{f}(\tau)$, see equation $\left.(11)\right)$ and the projection of the nonlinear forces (i.e. $\tilde{\mathbf{C}}_{0}^{f n l}(\tau), \tilde{\mathbf{C}}_{j}^{f n l}(\tau)$ and $\tilde{\mathbf{S}}_{j}^{f n l}(\tau)$ with $j=1, \cdots, m$, see equation (12)).

The expressions of $\tilde{\mathbf{I 0}}(\tau), \tilde{\mathbf{I}}(\tau)$ and $\tilde{\mathbf{I}}(\tau)$ are given by

$$
\begin{gathered}
\tilde{\mathbf{I 0}}(\tau)=\operatorname{diag}\left(\tilde{\mathbf{K}}(\tau), \mathbf{I} \tilde{\mathbf{0}}_{1}(\tau), \ldots, \mathbf{I} \tilde{\mathbf{0}}_{j}(\tau), \ldots, \mathbf{\mathbf { I }} \tilde{\mathbf{0}}_{m}(\tau)\right) \\
\tilde{\mathbf{I}}(\tau)=\operatorname{diag}\left(\mathbf{0}, \mathbf{I} \tilde{\mathbf{1}}_{1}(\tau), \ldots, \mathbf{\mathbf { I }} \tilde{\mathbf{1}}_{j}(\tau), \ldots, \mathbf{I} \tilde{\mathbf{1}}_{m}(\tau)\right) \\
\tilde{\mathbf{I}}(\tau)=\operatorname{diag}\left(\mathbf{0}, \mathbf{I} \tilde{\mathbf{2}}_{1}(\tau), \ldots, \mathbf{I} \tilde{\mathbf{2}}_{j}(\tau), \ldots, \mathbf{I} \tilde{\mathbf{2}}_{m}(\tau)\right)
\end{gathered}
$$

with

$$
\begin{gathered}
\tilde{\mathbf{0}}_{j}(\tau)=\left[\begin{array}{cc}
\tilde{\mathbf{K}}(\tau) & \mathbf{0} \\
\mathbf{0} & \tilde{\mathbf{K}}(\tau)
\end{array}\right] \\
\tilde{\mathbf{1}}_{j}(\tau)=\left[\begin{array}{cc}
\mathbf{0} & j \tilde{\mathbf{C}}(\tau) \\
-j \tilde{\mathbf{C}}(\tau) & \mathbf{0}
\end{array}\right] \\
\tilde{\mathbf{I}}_{j}(\tau)=\left[\begin{array}{cc}
-j^{2} \tilde{\mathbf{M}}(\tau) & j \tilde{\mathbf{G}}(\tau) \\
-j \tilde{\mathbf{G}}(\tau) & -j^{2} \tilde{\mathbf{M}}(\tau)
\end{array}\right]
\end{gathered}
$$


At this stage, we propose to expand the uncertain parameters by using the classical Karhunen-Loeve expansion [23] with the Galerkin formulation of the finite element method [7] as following

$$
\tilde{\mathbf{Z}}(\tau)=\overline{\mathbf{Z}}+\sum_{l=1}^{L} \xi_{l}(\tau) \mathbf{Z}_{l}
$$

where $\tilde{\mathbf{Z}}(\tau)$ denotes one of the possible uncertain quantities. $\overline{\mathbf{Z}}$ is the mean of quantity $\tilde{\mathbf{Z}}$ and $\mathbf{Z}_{l}$ designates the $l$ th term of the Karhunen-Loeve expansion. We draw attention to the fact that in the presence of several random quantities we have several independent Gaussian random variables $\xi_{l}(\tau)$ for each uncertain quantity.

Furthermore the stochastic dynamic response $\tilde{\mathbf{X}}(\tau)$ is expanded on the Polynomial Chaos basis [7, 24] :

$$
\tilde{\mathbf{X}}(\tau)=\sum_{j=0}^{\infty} \mathbf{X}_{j} \Psi_{j}(\underline{\xi}(\tau))
$$

where $\Psi_{j}(\underline{\xi}(\tau))$ designates the polynomials of the basis with $\underline{\xi}(\tau)$ the random vector and $\mathbf{X}_{j}$ the deterministic coefficient to be found. In this study, the uncertain parameters will follow the Gaussian law, consequently the polynomials considered are the Hermite polynomials. A truncated form to a finite number $N_{p}$ of the previous expression (21) is given by

$$
\tilde{\mathbf{X}}(\tau)=\sum_{j=0}^{N p} \mathbf{X}_{j} \Psi_{j}(\underline{\xi}(\tau))
$$

where $N_{p}$ defines the number of polynomials given by $N p=\frac{(p+r) !}{p ! r !}$ where $r$ is the number of random variables and $p$ is the chaos order.

The Karhunen-Loeve expansion for all material quantities and for forces coefficients, given by Equation (20), can be rewritten, when considering a more global quantity $\bullet_{j}\left(\operatorname{as}_{\mathbf{I}} \mathbf{0}_{j}, \mathbf{I} \mathbf{1}_{j}, \mathbf{I} \mathbf{2}_{j}, \mathbf{F}_{j}\right.$ and $\mathbf{F}_{\mathbf{n l}}$ ), and identified on the whole basis defined by vector $\xi(\tau)$, as

$$
\tilde{\bullet}_{j}(\tau)=\sum_{j=0}^{\infty} \bullet_{j} \Psi_{j}(\underline{\xi}(\tau)),
$$

Quantity $\bullet_{j}$ will be detailed in the next sections for each uncertain quantity of the problem.

As previously explained, the frequency parameter needs also to be consider as a function of the random parameter in the context of solving a nonlinear problem with uncertainties. So we propose to expand the stochastic frequency $\tilde{\omega}(\tau)$ on the chaos basis by considering the following form

$$
\tilde{\omega}(\tau)=\sum_{j=0}^{N h} \omega_{j} \Psi_{j}(\underline{\xi}(\tau))
$$

where $\omega_{j}$ are the deterministic coefficients to find. $N h$ defines the number of polynomials (i.e. $N h=\frac{(h+r) !}{h ! r !}$ where $r$ is the number of random variables and $h$ is the chaos order for the decomposition of the stochastic frequency). For the sake of simplicity, $N h$ can be chosen to be equal to $N p$.

By substituting Equation (22) to Equation (24) in Equation (13) and after projection on $\left\{\Psi_{m}\right\}_{m=0}^{N p}$ basis, the following nonlinear expression can be found [13]:

$$
\hat{\mathbf{L}}\left(\omega_{j}\right) \hat{\mathbf{X}}=\hat{\mathbf{F}}+\hat{\mathbf{F}}_{\mathbf{n l}}(\hat{\mathbf{X}})
$$

where $\omega_{j}$ (for $j=0, \ldots, N_{p}$ ), are the unknown deterministic coefficients of the stochastic frequency $\tilde{\omega}(\tau)$ (see equation $(24)$ ). As indicated in [13], $\hat{\mathbf{L}}$ includes contributions from linear and quadratic terms of $\omega_{i}$. The size of the previous nonlinear equation $(25)$ is $(2 m+1) \times N p \times n_{D O F}$ with $(2 m+2) \times N p \times n_{D O F}$ unknowns where $n_{D O F}$ defines the number of degree-of-freedom 
for the original deterministic rotor system. For the interested reader, all the calculations and expressions of $\hat{\mathbf{L}}, \hat{\mathbf{F}}, \hat{\mathbf{F}}$ nl and $\hat{\mathbf{X}}$ are given [13].

The non linear stochastic term $\hat{\mathbf{F}}_{\mathbf{n l}}$ can be evaluated via an Alternate Frequency-Time method with Probabilistic Collocation (AFT-PC method [13]). The Probabilistic Collocation uses a non-intrusive approach to compute the polynomial chaos description of uncertainty numerically at collocation points in probability space [25]. First of all, by considering the stochastic dynamic response of the nonlinear system $\tilde{\mathbf{X}}(\tau)$ and using $n$ Gauss locations with an Inverse Discrete Fourier Transform (IDFT) procedure, one can evaluate the harmonic components of the response $\{\mathbf{X}\}_{n}$ in the frequency domain and the associated displacements $\{\mathbf{x}(t)\}_{n}$ in the time-domain (for the reader comprehension, the notation $\mathbf{x}(t)$ represents the deterministic truncated Fourier series of $\tilde{\mathbf{x}}(\tau, t)$ given in equation (10)). Knowing the expression of nonlinear forces in time-domain, the associated nonlinear expressions $\left\{\mathbf{f}_{\mathbf{n l}}(t)\right\}_{n}$ can be easily calculated where $\mathbf{f}_{\mathbf{n l}}(t)$ defines the deterministic truncated Fourier series of $\tilde{\mathbf{f}_{\mathbf{n l}}}(\tau, t)$ given in equation (12). Then, the calculation of the harmonic components $\left\{\mathbf{F}_{\mathbf{n l}}\right\}_{n}$ and the random vector $\tilde{\mathbf{F}}_{\mathbf{n l}}(\tau)$ of the nonlinear forces in the frequency domain can be obtained by using the Discrete Fourier Transform (DFT) and by the expansion on the chaos basis. The general algorithm procedure for the Alternate Frequency-Time method with Probabilistic Collocation can be schematically given by

$$
\begin{aligned}
& \tilde{\mathbf{X}}(\tau)=\sum_{j=0}^{N p} \mathbf{X}_{j} \Psi_{j}(\underline{\xi}(\tau)) \quad \underset{n \text { Gauss locations }}{\stackrel{\text { evaluation }}{\longrightarrow}}\{\mathbf{X}\}_{n} \stackrel{\text { IDFT }}{\longrightarrow}\{\mathbf{x}(t)\}_{n} \\
& \tilde{\mathbf{F}}_{\mathbf{n l}}(\tau)=\sum_{j=0}^{N p} \mathbf{F}_{\mathbf{n l} j} \Psi_{j}(\underline{\xi}(\tau)) \stackrel{\text { projection on } \Psi_{j}}{\longleftarrow} \quad\left\{\mathbf{F}_{\mathbf{n l}}\right\}_{n} \stackrel{\text { DFT }}{\longleftarrow}\left\{\begin{array}{c}
\downarrow \\
\left.\mathbf{f}_{\mathbf{n l}}(t)\right\}_{n}
\end{array}\right.
\end{aligned}
$$

For the interested reader, it can be noted that the AFT-PC method is performed for a given value of the stochastic frequency due to the fact that this process is performed only for estimating the random vector of the nonlinear forces $\tilde{\mathbf{F}}_{\mathbf{n l}}(\tau)$ for an initial estimation of the stochastic nonlinear response $\tilde{\mathbf{X}}(\tau)$. The process of iteration on the unknowns of the problem is achieved via equation 25. Generally, predictor and corrector processes are also applied to track the evolution of the system behavior for different operational points of interest as one of its parameter varies. In the present study, we consider the evolution of the rotational speed (with the possibility of an adapted step according to changes of the amplitudes of non-linear responses) and the tangent and arc-length methods [13] are used as predictor and corrector for the Stochastic Harmonic Balance Method. For the calculations performed via the Monte-Carlo simulations, the same predictor and corrector processes will be used.

\section{Numerical results}

In this section, the effects of uncertainties on the rotor system will be investigated. Two specific rotor systems will be considered: the first one concerns the rotor with a cubic non-linear stiffness as previously indicated in equation (2). The second case is the rotor with the contact/non-contact element as previously defined in equation (3).

Table 1 gives the usual deterministic parameters for the rotor system in all cases. In addition to the deterministic formulation, uncertainties on the different structural parameters, linear or nonlinear forces are investigated. Table 2 summarizes the values of the uncertain parameters for the different cases (hypotheses for each case: dispersion is taken around the mean equal to the deterministic value given in Table 1). Characteristics used in the computations (for chaos and HMB orders) are also given in Table 2. It should be noted that the samples of the Gaussian law have been checked to be all positive for all cases computed.

For all cases under study and the drawing of the stochastic nonlinear responses via the Frequency Response Functions, a deterministic nonlinear response (black lines) will be firstly shown. These results are based on a classical deterministic procedure (via the deterministic Harmonic Balance Method) by using the deterministic values of the physical parameters. 
To validate the Stochastic Harmonic Balance Method, all the calculations are compared to those obtained from the MonteCarlo Simulations. Thereby, results of the Monte Carlo simulations (plots in grey) are only based on the deterministic Harmonic Balance Method (with a deterministic frequency). $n b_{M C}$ samples of the random vector $\{\underline{\xi}(\tau)\}_{i}\left(\right.$ for $\left.i=1, \ldots, n b_{M C}\right)$ are considered. The associated nonlinear responses represent the reference solutions $\{\mathbf{X}\}_{i(\mathrm{MCS}}$ ) (for $i=1, \ldots, n b_{M C}$ ). Regarding the results via the Stochastic Harmonic Balance Method (plots in red), the latter are obtained by performing the previous computational process with the stochastic dynamic response and the stochastic frequency (see Section 3). In order to be able to compare for all the $n b_{M C}$ samples the nonlinear solutions via the Monte Carlo simulation (denoted $\left.\{\mathbf{X}\}_{i(\mathrm{MCS}}\right)$ for $\left.i=1, \ldots, n b_{M C}\right)$ and the Stochastic Harmonic Balance Method (denoted $\mathbf{X}_{i}$ for $i=1, \ldots, n b_{M C}$ ), the same samples $\{\underline{\xi}(\tau)\}_{i}$ have to be used for the evaluation of Hermite polynomials. The computational procedure for the Monte Carlo simulations and the Stochastic Harmonic Balance Method is illustrated in Figure 2.

All the results detailed in the new paragraphs are presented at $z=0.2 m$ in the $y$-direction for a rotor frequency evolution from $5 \mathrm{~Hz}$ to $275 \mathrm{~Hz}$ (with an adaptive step, as indicated in the previous section).

[Figure 2 about here.]

[Table 1 about here.]

[Table 2 about here.]

\subsection{Rotor system with the cubic nonlinear stiffness}

\subsubsection{Cases 1 and 2: uncertainties in the Young modulus}

First of all, uncertainties on the Young modulus are investigated. Considering the previous equation 5, the variation coefficient on the Young modulus is taken as $\delta_{E}=2.5 \%$ (case 1) or $\delta_{E}=5 \%$ (case 2). Figures 3 (a) and (b) present the frequency response functions for the stochastic non-linear response for both the MCS and the Stochastic-HBM.

First of all, it is observed that the random parameter on the Young modulus affects the nonlinear responses at the three critical speeds. Obviously it appears that increasing the value of the dispersion on Young modulus increases the variability on the frequency response functions of the non-linear rotor system. Showing more specifically the third critical speed (around $250 \mathrm{~Hz}$ ), we can see that the stochastic dynamic response keeps an envelope having the shape of a typical hardening non-linear behavior.

Then, the numerical results show that both the magnitudes of the nonlinear rotor response and the values of the critical speed of the flexible rotor are affected by the random variable $\tilde{E}$. Thus, the uncertainty introduced in the Young modulus induces an important variability on the critical speeds: for example, the values of the third critical speed are changed over $20 \mathrm{~Hz}$ for case 2 . We recall that the hardening nonlinear behavior is due to the presence of the nonlinear cubic term given in Equation (2).

Finally, as indicated in Figures 3 (a) and (b), the response of the nonlinear system is not unique for some excitation frequencies (see more specifically the frequency range in the intervals $[250 ; 260] \mathrm{Hz}$ ). We can see that three response levels with low amplitude or possible high amplitude can be detected in the vicinity of the initial conditions. As previously mentioned in [13], these sensitivities with respect to a linear random variable on the dynamic behavior of a mechanical nonlinear system is one of the most difficult aspects to be captured by uncertainty methods (i.e. capability to solve problems including returning points in order to describe multi-solutions as usually observed for nonlinear systems). In the present case, the Stochastic-HBM approach proves to be an useful tool due to the fact that the pulsation frequency is considered to be random, as explained in the previous section. 
By performing Monte Carlo simulations, the results obtained via the Stochastic-HBM with the Alternate Frequency-Time method with Probabilistic Collocation appears to agree very well. So for the design of rotating machines, the presented procedure based on the Stochastic-HBM seems to be a useful tool in order to give valuable information regarding the degree of the influence of nonlinear elements and random parameters.

[Figure 3 about here.]

\subsubsection{Case 3: uncertainty in the unbalance force}

In this part of the paper, we consider the uncertainty present on the unbalance forces, as in equation 8 . The variation coefficient on the unbalance excitation is taken as $\delta_{m}=5 \%$ (case 3). Figure 4 gives the frequency response function for the stochastic non-linear response of the flexible rotor system.

We note a global dependency in the value of the critical speeds and the vibration levels of the nonlinear response versus the random parameter. In comparison with the previous cases (cases 1 and 2), a strong sensitivity to the amplitudes of the flexible rotor and a high level of uncertainty are observed for the three critical speeds due to the introduction of the random parameter. This is easily explained by the fact that the random parameter relates to the excitation linear force (i.e. the unbalanced contribution of the rotor system). Moreover, it is interesting to note that the uncertainty on the unbalance forces also influences the nonlinear hardening behavior of the rotor system: showing the evolution of the nonlinear stochastic response around the three critical speeds, it is more specifically observed an important variation of the hardening nonlinear behavior. We recall that the variation of the unbalanced forces drastically influences the amplitudes of the nonlinear response and so increases or decreases the appearances of the hardening behavior of the frequency response function at the critical speeds. This fact has been previously shown and explained in details in [13].

It can be concluded that, even if the value of the random parameter $\delta_{m}$ remains low, this uncertainty introduced in the unbalanced linear forces of the rotor system represents an important aspect to be considered during the design of a rotating machines.

Concerning the validity of the Stochastic-HBM, the remarks previously stated remain valid: the MCS and the StochasticHBM are in perfect agreement.

[Figure 4 about here.]

\subsubsection{Case 4: uncertainty in the nonlinear cubic stiffness}

In this part, we are interested in the effect of variations on the cubic stiffness. The variation coefficient on the nonlinear cubic stiffness is taken as $\delta_{N L}=10 \%$ (case 4). Figure 5 presents the results obtained by the Monte-Carlo Simulations and the Stochastic Harmonic Balance Method for the frequency range of interest.

Due to the variability of the cubic stiffness, the Frequency Response Function shows a more or less pronounced curved peak frequency and a more or less abrupt turning point, as illustrated for the third critical speed (see more specifically the frequency range in the intervals $[250 ; 260] \mathrm{Hz}$ ). It is recalled that the presence of uncertainty on the nonlinear cubic stiffness can increase or decrease the hardening behavior of the mechanical system.

Moreover, it is observed that the vibration amplitudes can drastically change due the variability of the cubic stiffness. The maximum level is obtained when the hardening behavior is most marked (see Figure 5 around $260 \mathrm{~Hz}$ ). This result is significantly different from the previous results for cases 1 and 2 where the maximum level was similar along the frequency range in the 
intervals $[240 ; 260] \mathrm{Hz}$ (see Figure 3(b)). These results demonstrate that uncertainties introduced in linear or nonlinear part of the rotor system can substantially modify the nonlinear stochastic response and thus the choice of the design variables that need to be consider as uncertain parameters is a key point to be investigated during the design process of a flexible rotor system.

Again a perfect correlation between the Stochastic-HBM and MCS results is observed even if the random parameter is introduced on the nonlinear terms inducing a strong evolution of the hardening effect of the rotor system. These results clearly demonstrate the efficiency of the Stochastic-HBM and the need to consider uncertainty of the nonlinear elements of rotating machines in order to avoid improper design.

[Figure 5 about here.]

\subsubsection{Case 5: uncertainties for both the Young modulus and the nonlinear cubic stiffness}

Now we treat the case in which uncertain quantities come from two parameters studied previously: case 5 concerns a variation on the Young modulus (i.e. $\delta_{E}=2.5 \%$ ) and a variation in the nonlinear cubic stiffness (i.e. $\delta_{N L}=10 \%$ ). As previously explained, it was observed that including uncertainty on one or the other of these two parameters changes the response of the rotor system. So, it is interesting to undertake the stochastic nonlinear response if uncertainties are present on both parameters. Figure 6 illustrates the frequency function response. First, it is observed that the evolution of the curvature of the resonance peak around the third critical speed is still pronounced (due the variability of the nonlinear cubic stiffness). Secondly, the range of variation increases in comparison with the previous case 4 due to the addition of random Young modulus. Again these results demonstrate that the choice of the random parameters is essential and that the combination of design variables as random parameters has to be done in order to lead to a relevant design for a flexible rotor system. It is also shown again that the response of the nonlinear system is not unique for some excitation frequencies. Over a range of frequencies near the resonance, the system can take one of three possible response levels.

Finally, a very good agreement is obtained between the results from the MCS and the Stochastic-HBM demonstrating the efficiency of the Stochastic-HBM even if several random parameters are used in the design process.

[Figure 6 about here.]

\subsection{Rotor system with the contact/non-contact behavior}

In this section, the stochastic response of the rotor system with the contact/non-contact behavior (see equation 3) will be undertaken. The targets are twofold: the validation of the Stochastic-HBM for a nonlinear system with a non-regular nonlinearity and the analysis of the behavior of the flexible system including uncertainties. Two specific cases will be detailed: the first one (case 6) concerns the possibility of uncertainty on Young modulus (i.e. linear part of the flexible rotor) and the second case (case 7) corresponds to the flexible rotor with uncertainty on the effective stiffness of the system in contact state (the nonlinearity of contact and no-contact states is supposed to be random by considering the random parameter $\tilde{k_{2}}$ ).

\subsubsection{Case 6 : uncertainty on the Young modulus}

First, we are interested in the effect of variations on Young modulus (case 6 Table 1) for the rotor system with a non-regular nonlinearity (i.e. deterministic contact/non-contact state at the first support of the rotor). The variation coefficient on Young modulus is taken as $\delta_{E}=5 \%$ (case 6). Figure 7 shows the frequency response function for the stochastic non-linear response for both the MCS and the Stochastic-HBM. In the vicinity of the second critical speed (around $[100 ; 120] \mathrm{Hz}$ ), the classical behavior 
and characteristic of nonlinear systems with contact are observed: due to the transition between the non contact state and contact state, a break of slope of the peak is shown. It can be noticed that both the deterministic nonlinear stiffness and the uncertainty introduced in the Young modulus result in a significant change in the evolution of the curvature of the second resonance peak of the flexible rotor. As previously seen for cases 1 and 2, the variation of the Young modulus induces a variability of the magnitude of the nonlinear response resonance frequency and a shift of the resonance peaks.

\subsubsection{Case $7:$ uncertainty on the effective stiffness in contact state}

For the last case, we consider the variation coefficient of the effective stiffness $\delta_{k 2}=10 \%$ (case 7). Figure 8 presents the results obtained by the MCS and the Stochastic-HBM for the frequency range of interest. It is interesting to see that the variability of the effective stiffness can drastically change not only the magnitudes of the nonlinear response but also the hardening behavior of the flexible rotor. Indeed, the Frequency Response Function shows a more or less pronounced curved peak for the second critical speed with a more or less abrupt turning point. Then, the random parameter greatly increases the frequency range where the maximum level is obtained for the third critical speed (see Figure 8 in the intervals $[240 ; 270] \mathrm{Hz}$ ). As previously seen for case 4, the maximum level for each peak of the stochastic response corresponds to the maximum potential of curvature for the system (i.e. for the most marked hardening behavior, see Figure 8 around $270 \mathrm{~Hz}$ for example).

Finally, all these results for cases 6 and 7 are in agreement with the previous remarks and conclusions: uncertainties introduced in linear or nonlinear parts of the system can cause major changes and modifications in the nonlinear stochastic response. Moreover, the efficiency of the Stochastic-HBM for a flexible rotor with non-regular nonlinearity is clearly demonstrated.

[Figure 7 about here.]

[Figure 8 about here.]

\section{Conclusion}

In this paper the Stochastic Harmonic Balance Method has been developed to efficiently compute nonlinear response amplitudes for a flexible rotor system with local nonlinearities. The efficiency of the Stochastic-HBM is validated by performing Monte Carlo simulations. The uncertainties are introduced in the linear and nonlinear design variables that characterize the flexible rotor system.

The effect of different linear and nonlinear random variables on the dynamic behavior of the flexible rotor was examined in details. It was clearly illustrated that introducing uncertainties in the design process is recommended in order to optimize the design.

Thus, the proposed Stochastic Harmonic Balance Method appears to be a robust and useful tool for the design and analysis of flexible rotor systems with uncertainties and local nonlinearities. This methodology allows the adaptation of stochastic representations to a new class of problem of interest in rotor dynamics or other dynamics problems. However, many open questions have to be solved in the future. From a practical point of view, one of the most crucial next step can be to be able to propose more practical and commonly implemented stochastic nonlinear techniques for the reliability and safety of complex engineering structures.

At last, one of the current limitations of the proposed method lies in the size of the nonlinear systems considered (i.e. the number of degrees of freedom) and the size of the polynomial chaos basis to consider. Future research should also be undertaken on these points 


\section{References}

[1] D. Childs. Turbomachinery Rotordynamics: Phenomena, Modeling, and Analysis. Wiley Interscience, 1993.

[2] T. Yamamoto and Y. Ishida. Linear and Nonlinear Rotordynamics: A Modern Treatment with Applications. Wiley and Sons, 2001.

[3] M. Friswell, J.E.T. Penny, S.D. Garvey, and A.W. Lees. Dynamics of rotating machines. Wiley and Sons, 2010.

[4] A. Nayfeh. Perturbation Methods. John Wiley and Sons, London, 1973.

[5] H. Benaroya and M. Rehak. Finite element methods in probabilistic structural analysis: A selective review. Applied Mechanics Reviews, 41(5):201-213, 1988.

[6] F. Yamazaki, M. Shinozuka, and G. Dasgupta. Neumann expansion for stochastic finite element analysis. Journal of Engineering Mechanics, ASCE, 114(8):1335-1354, 1988.

[7] R. Ghanem and P. Spanos. Stochastic Finite Elements: A Spectral Approach. Springer-Verlag, 1991.

[8] R. Ghanem. Stochastic finite elements with multiple random non- gaussian properties. Journal of Engineering Mechanics, 60(4):26-40, 1999.

[9] J. Didier, J-J. Sinou, and B. Faverjon. Multi-dimensional harmonic balance with uncertainties applied to rotor dynamics. Journal of Vibration and Acoustic, 134(6):061003, 17 pages, 2012.

[10] J. Didier, B. Faverjon, and J-J. Sinou. Analyzing the dynamic response of a rotor system under uncertain parameters by polynomial chaos expansion. Journal of Vibration and Control, 18(5):587-607, 2012.

[11] E.H. Koroishi, A.A. Cavalini, A.M.G. de Lima, and V. Steffen. stochastic modeling of flexible rotors. Journal of the Brazilian Society of Mechanical Sciences and Engineering, 34(2):574-583, 2012.

[12] J. Didier, J-J. Sinou, and B. Faverjon. Study of the non-linear dynamic response of a rotor system with faults and uncertainties. Journal of Sound and Vibration, 331:671-703, 2012.

[13] J. Didier, J-J. Sinou, and B. Faverjon. Nonlinear vibrations of a mechanical system with non-regular nonlinearities and uncertainties. Communications in Nonlinear Science and Numerical Simulation, 18(11):3250-3270, 2013.

[14] Y-B. Kim and Choi S-K. A multiple harmonic balance method for the internal resonant vibration of a non-linear jeffcott rotor. Journal of Sound and Vibration, 208(3):745-761, 1997.

[15] B. Cochelin and Vergez C. A high order purely frequency-based harmonic balance formulation for continuation of periodic solutions. Journal of Sound and Vibration, 324(1-2):243-262, 200ç.

[16] J-J. Sinou. Non-linear dynamics and contacts of an unbalanced flexible rotor supported on ball bearings. Mechanism and Machine Theory, 44:1713-1732, 2009.

[17] V. Jaumouille, J-J. Sinou, and B. Petitjean. An adaptive harmonic balance method for predicting the nonlinear dynamic responses of mechanical systems - application to bolted structures. Journal of Sound and Vibration, 329(19):4048-4067, 2010 . 
[18] V. Jaumouille, J-J. Sinou, and B. Petitjean. Simulation of payne effect of elastomeric isolators with a harmonic balance method. Shock and Vibration, 19:1281-1295, 2012.

[19] S. Karkar, B. Cochelin, and Vergez C. A high-order, purely frequency based harmonic balance formulation for continuation of periodic solutions: The case of non-polynomial nonlinearities. Journal of Sound and Vibration, 332(4):968-977, 2013.

[20] T. M. Cameron and J. H. Griffin. An alternating Frequency/Time domain method for calculating the Steady-State response of nonlinear dynamic systems. Journal of Applied Mechanics, 56(1):149-154, March 1989.

[21] F.E. Erich. Handbook of Rotordynamics. McGraw-Hill, 1992.

[22] J-J. Sinou and B. Faverjon. The vibration signature of chordal cracks in a rotor system including uncertainties. Journal of Sound and Vibration, 331:138-154, 2012.

[23] M. Loève. Probability Theory, $4^{\text {th }}$ edition. Springer-Verlag, 1977.

[24] N. Wiener. The homogeneous chaos. American Journal of Mathematics, 60(4):897-936, 1938.

[25] J.A.S. Witteveen, A. Loeven, S. Sarkar, and H. Bijl. Probabilistic collocation for period-1 limit cycle oscillations. Journal of Sound and Vibration, 311(1-2):421-439, 2008. 


\section{List of Figures}

1 Finite-element model of the rotor system (a) rotor system (b) coordinates in fixed frame . . . . . . . . . . 15

2 Computational procedure for the estimation of the Frequency Response Functions via the Monte Carlo simulations and the Stochastic Harmonic Balance Method . . . . . . . . . . . . . . . . . . . 16

3 Frequency Response Functions of the random nonlinear response for cases 1 and 2, (a) case 1 (b) case 2 - MCS (plots in grey), Stochastic-HBM (plots in red) and deterministic response (black) $\ldots \ldots \ldots \ldots \ldots \ldots$

4 Frequency Response Functions of the random nonlinear response for case 3 - MCS (plots in grey), StochasticHBM (plots in red) and deterministic response (black) . . . . . . . . . . . . . . . . 18

5 Frequency Response Functions of the random nonlinear response for case 4 - MCS (plots in grey), StochasticHBM (plots in red) and deterministic response (black) . . . . . . . . . . . . . . . . . . . . . . 19

6 Frequency Response Functions of the random nonlinear response for case 5 - MCS (plots in grey), StochasticHBM (plots in red) and deterministic response (black) . . . . . . . . . . . . . . . . . 20

7 Frequency Response Functions of the random nonlinear response for case 6 - MCS (plots in grey), StochasticHBM (plots in red) and deterministic response (black) . . . . . . . . . . . . . . . . . . 21

8 Frequency Response Functions of the random nonlinear response for case 7 - MCS (plots in grey), StochasticHBM (plots in red) and deterministic response (black) . . . . . . . . . . . . . . . 22 


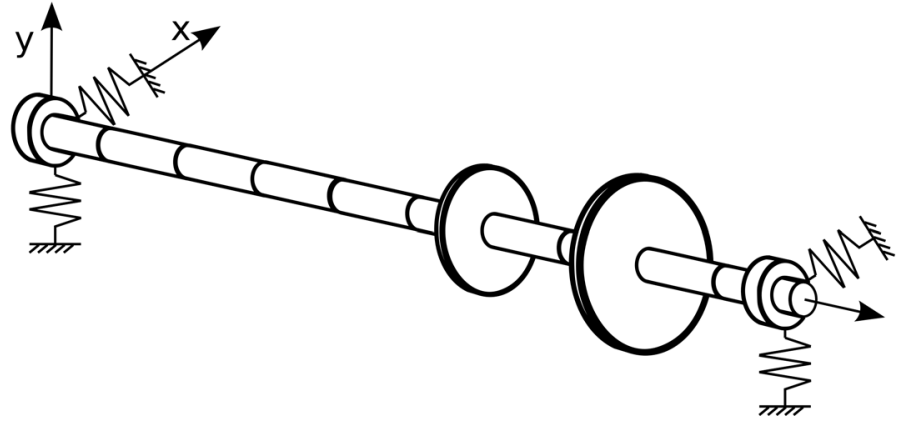

(a)

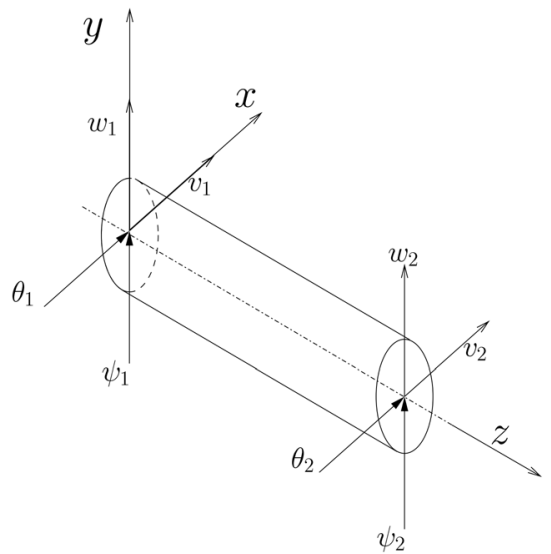

(b)

Fig. 1: Finite-element model of the rotor system (a) rotor system (b) coordinates in fixed frame 


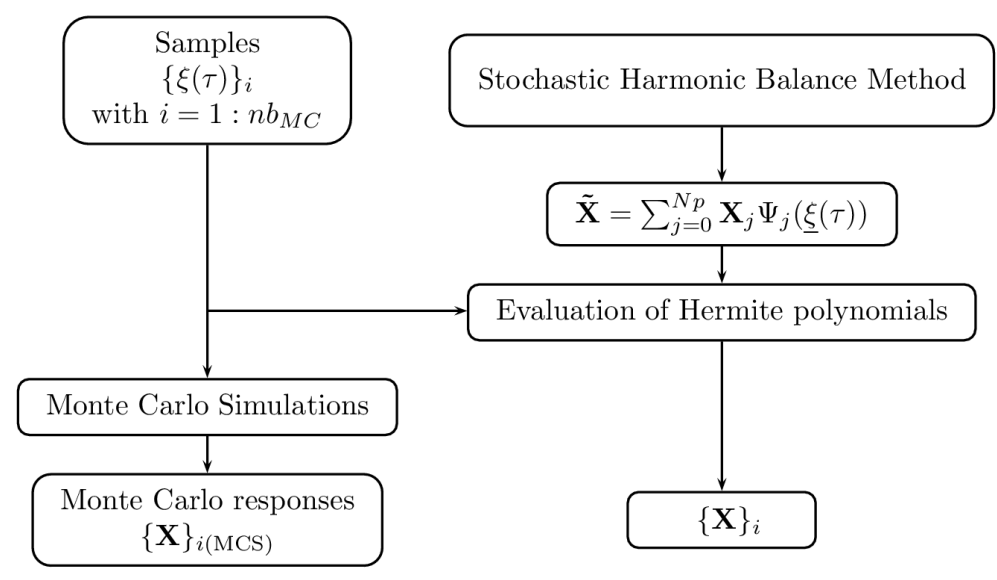

Fig. 2: Computational procedure for the estimation of the Frequency Response Functions via the Monte Carlo simulations and the Stochastic Harmonic Balance Method 


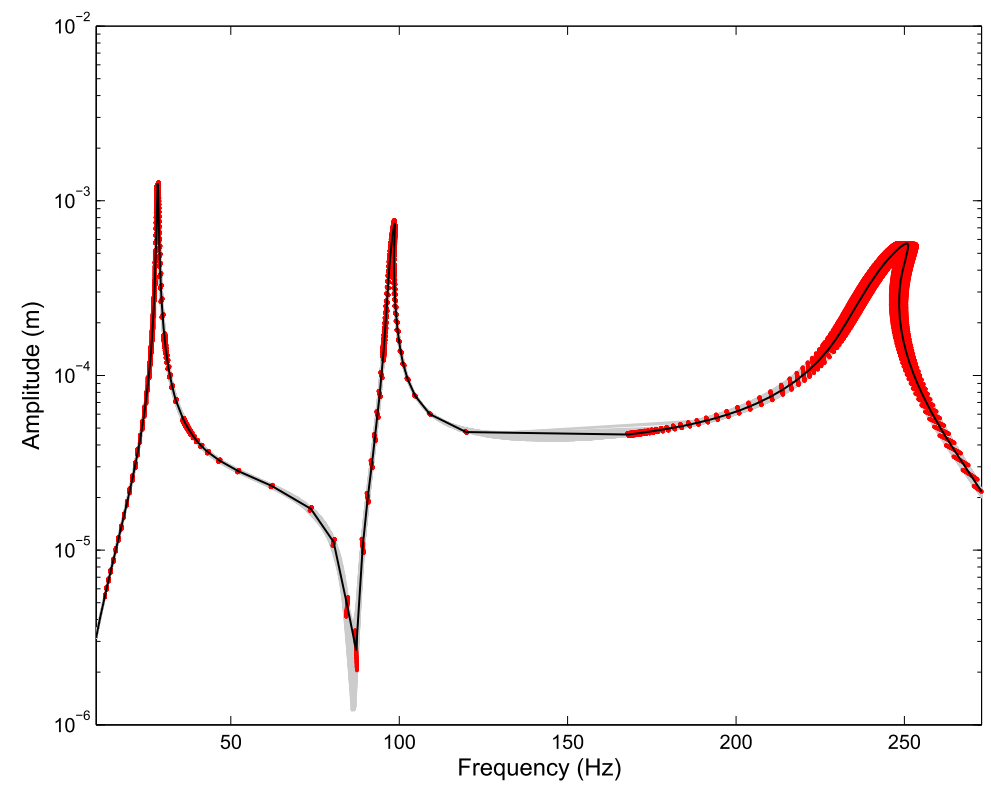

(a)

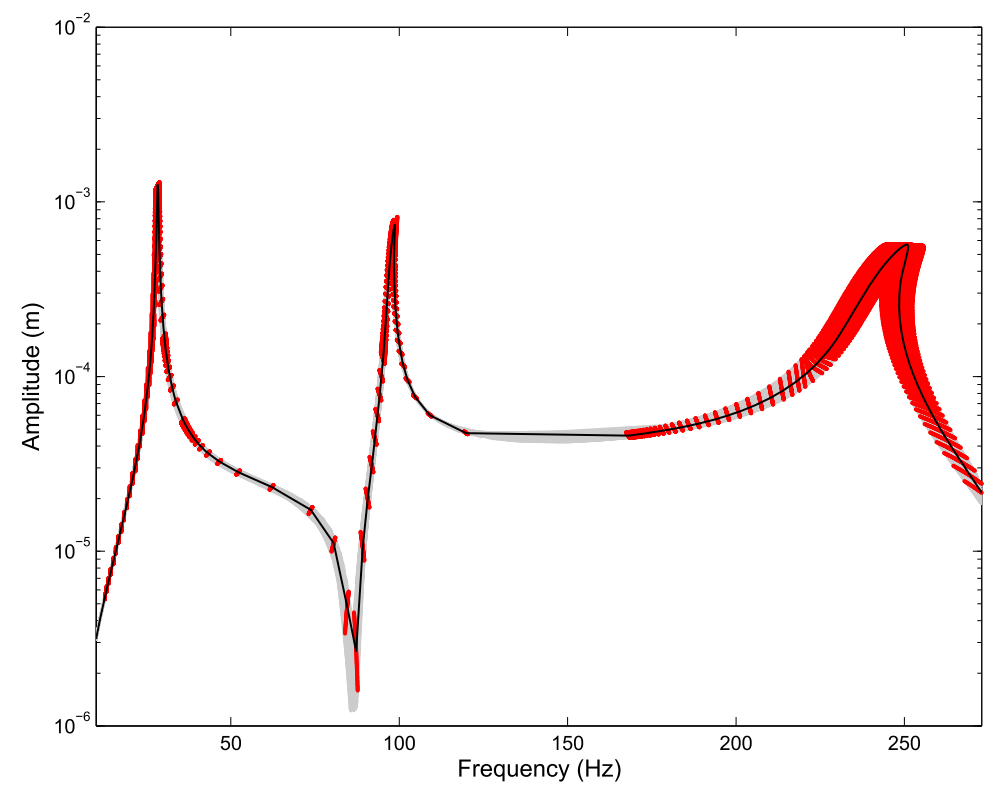

(b)

Fig. 3: Frequency Response Functions of the random nonlinear response for cases 1 and 2, (a) case 1 (b) case 2 - MCS (plots in grey), Stochastic-HBM (plots in red) and deterministic response (black) 


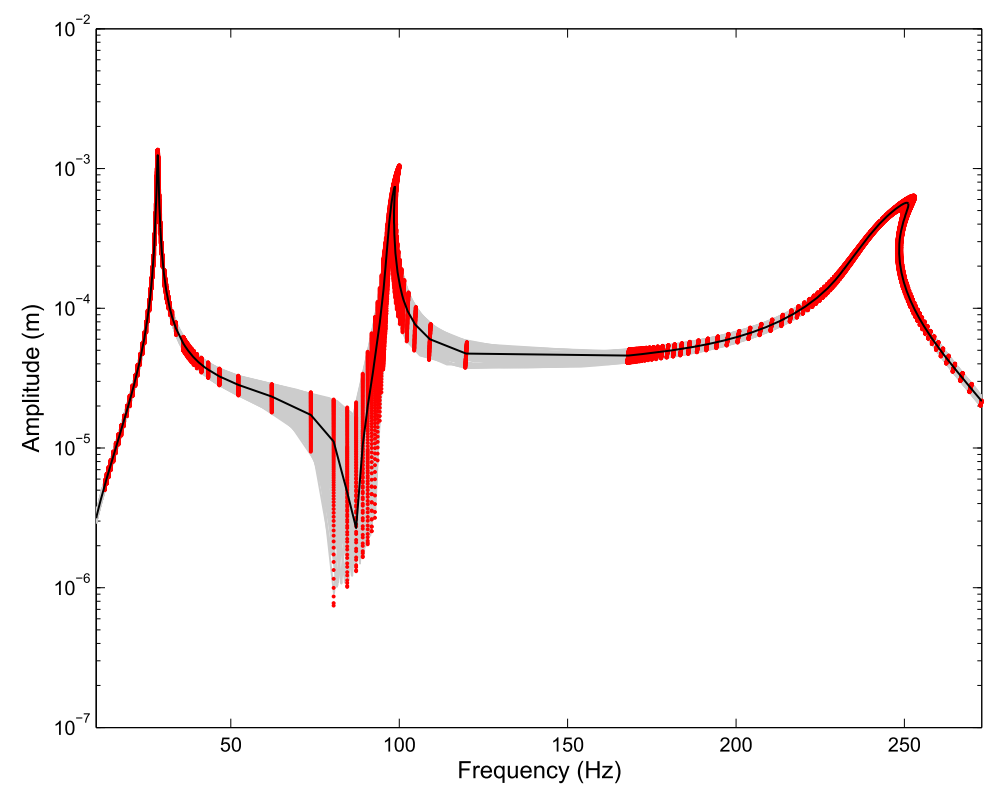

Fig. 4: Frequency Response Functions of the random nonlinear response for case 3 - MCS (plots in grey), Stochastic-HBM (plots in red) and deterministic response (black) 


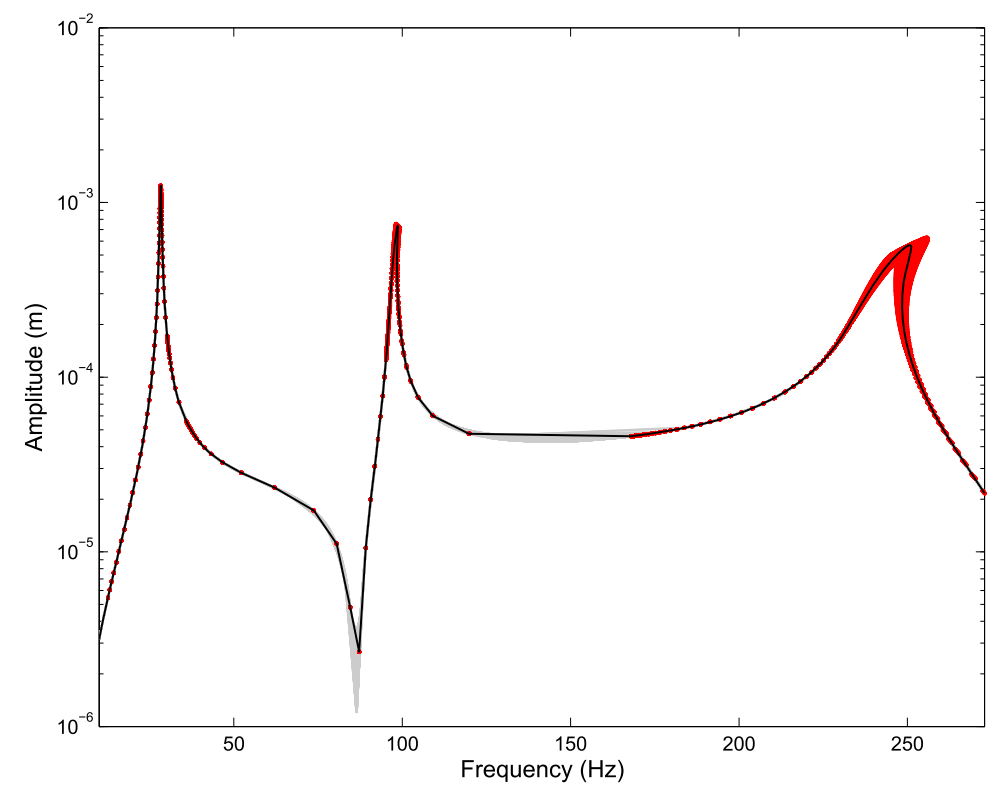

Fig. 5: Frequency Response Functions of the random nonlinear response for case 4 - MCS (plots in grey), Stochastic-HBM (plots in red) and deterministic response (black) 


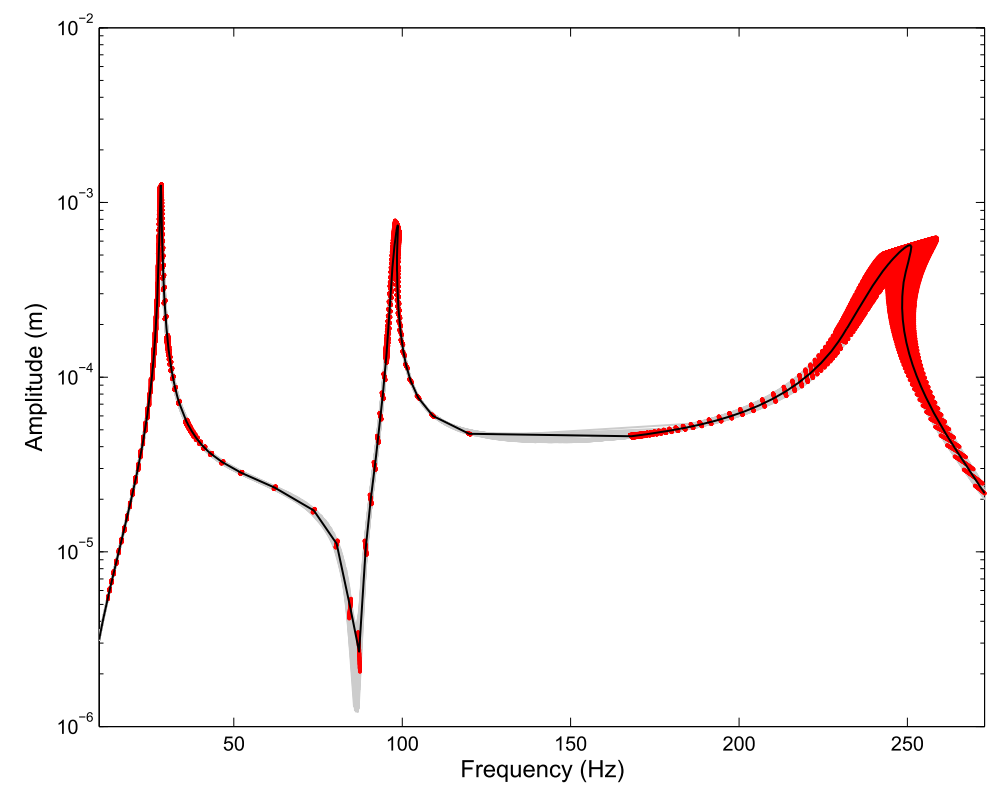

Fig. 6: Frequency Response Functions of the random nonlinear response for case 5 - MCS (plots in grey), Stochastic-HBM (plots in red) and deterministic response (black) 


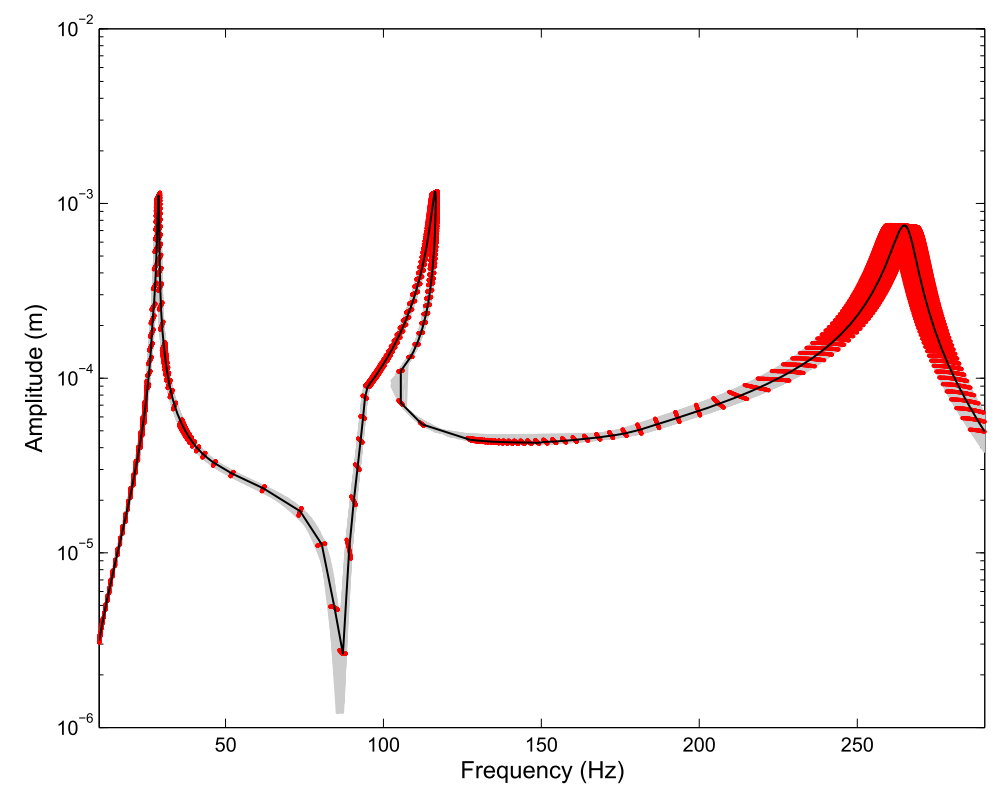

Fig. 7: Frequency Response Functions of the random nonlinear response for case 6 - MCS (plots in grey), Stochastic-HBM (plots in red) and deterministic response (black) 


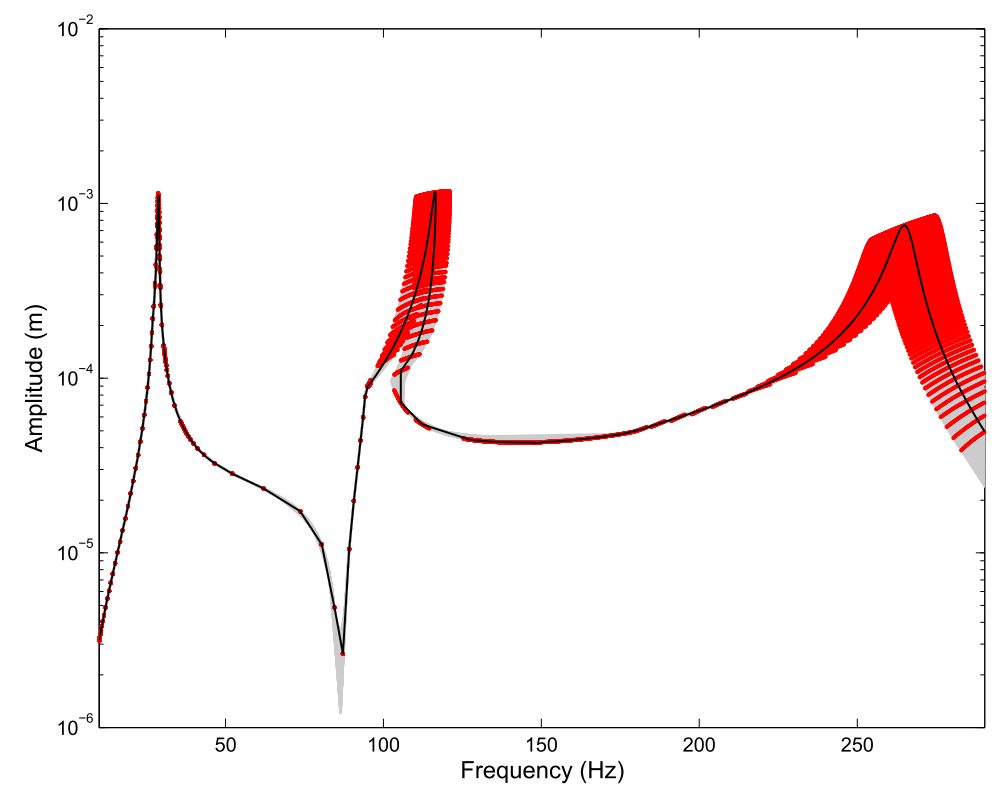

Fig. 8: Frequency Response Functions of the random nonlinear response for case 7 - MCS (plots in grey), Stochastic-HBM (plots in red) and deterministic response (black) 


\section{List of Tables}

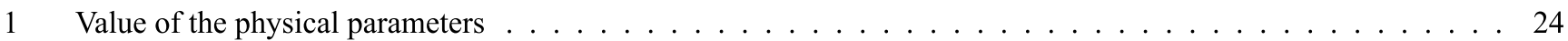

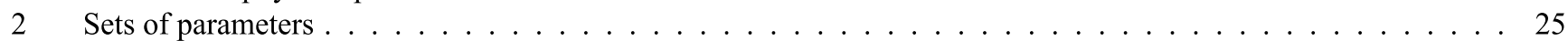




\begin{tabular}{cc}
\hline Description & Value \\
\hline Radius of the rotor shaft & $0.02 \mathrm{~m}$ \\
Length of the rotor shaft & $1 \mathrm{~m}$ \\
Position of disk 1 & $0.6 \mathrm{~m}$ \\
Outer radius of the disk 1 & $0.1 \mathrm{~m}$ \\
Thickness of the disk 1 & $0.02 \mathrm{~m}$ \\
Position of disk 2 & $0.8 \mathrm{~m}$ \\
Outer radius of the disk 2 & $0.2 \mathrm{~m}$ \\
Thickness of the disk 2 & $0.02 \mathrm{~m}$ \\
Young's modulus of elasticity & $2.110^{11} \mathrm{~N} . \mathrm{m}^{2}$ \\
Shear modulus & $810^{10} \mathrm{~N} . \mathrm{m}^{2}$ \\
Density & $7800 \mathrm{~kg} . \mathrm{m}^{-3}$ \\
Poisson ratio & 0.3 \\
Stiffness of supports & $10^{6} \mathrm{~N} . \mathrm{m}^{-1}$ \\
Mass unbalance & $0.0005 \mathrm{~kg}$ \\
Clearance value $r_{l i m}$ & $0.0001 \mathrm{~m}$ \\
Nonlinear stiffness $k_{n l}$ & $10^{11} \mathrm{~N} . \mathrm{m}^{-1 / 3}$ \\
stiffness $k_{2}$ & $10^{6} \mathrm{~N} . \mathrm{m}^{-1}$ \\
\hline
\end{tabular}

Tab. 1: Value of the physical parameters 


\begin{tabular}{llllllll}
\hline Case & Type & $\delta_{E}$ & $\delta_{k_{n l}}$ & $\delta_{k_{2}}$ & $\delta_{m}$ & Chaos order & HBM order \\
\hline 1 & $x^{3}$ & $2.5 \%$ & & & & 3 & 3 \\
2 & $x^{3}$ & $5 \%$ & & & $5 \%$ & 3 & 3 \\
3 & $x^{3}$ & & & & $5 \%$ & 3 \\
4 & $x^{3}$ & & $10 \%$ & & & 2 & 3 \\
5 & $x^{3}$ & $2.5 \%$ & $10 \%$ & & 3 & 1 \\
6 & contact & $5 \%$ & & \multirow{2}{*}{$10 \%$} & 3 & 1 \\
7 & contact & & & $10 \%$ & 3 & \\
\hline
\end{tabular}

Tab. 2: Sets of parameters 